\title{
Unidad didáctica multimedia para el abordaje de los temas de estadística en la modalidad de bachillerato por madurez utilizando la herramienta tecnológica eXeLearning
}

\section{Multimedia Instructional Unit for the Approach of Statistical Topics in the High School Diploma for Adults Program Using the eXeLearning Technological Tool}

\author{
Carlos Navarro* \\ Universidad Estatal a Distancia, San José, Costa Rica \\ ORCID: https://orcid.org/0000-0002-7272-0467 \\ Islande Delgado \\ Universidad Estatal a Distancia, San José, Costa Rica \\ ORCID: https://orcid.org/0000-0001-8344-7323 \\ María G. Calderón \\ Universidad Nacional de Costa Rica, Heredia, Costa Rica \\ ORCID: https://orcid.org/0000-0002-2877-8899
}

Recibido 07-08-18 Revisado 30-08-18 Aprobado 21-11-18 En línea 12-03-19

*Correspondencia

Email: cnavarro_zuru@hotmail.com
Citar como:

Navarro, C., Delgado, I., \& Calderón, M.G. (2019). Unidad didáctica multimedia para el abordaje de los temas de estadística en la modalidad de bachillerato por madurez utilizando la herramienta tecnológica eXeLearning. Propósitos y Representaciones, 7(2), 17-106. doi: http://dx.doi.org/10.20511/pyr2019.v7n2.229 


\section{Resumen}

En Costa Rica, antes del 2016 no se incluía el tema de estadística en las pruebas nacionales de bachillerato en la asignatura de Matemática. A partir de 2016, la prueba, compuesta por 60 ítems, incluye 9 ítems de estadística. Por otro lado, los maestros en sus lecciones usualmente utilizan métodos expositivos tradicionales, mediados por el uso de pizarra y materiales impresos. En este contexto, se evidenció la necesidad de crear e implementar nuevas técnicas de enseñanza en esta área, por lo que se elaboró y aplicó mediante la herramienta tecnológica eXeLearning una Unidad Didáctica Multimedia en Estadística. La unidad didáctica se aplicó a un grupo de 30 estudiantes de la modalidad bachillerato por madurez de la región de Turrialba en Costa Rica. Se utilizó un método cualitativo naturalista interpretativo fundamentado en la generación de categorías de análisis. Se determinó que una forma simple de mostrar la información y la inclusión de actividades de repaso, ejemplificación, práctica y evaluación, son deseadas y bien valoradas por los estudiantes. Además, se observó que el medio tecnológico promovió interés y motivación estudiantil, al tiempo que facilitó el seguimiento de las actividades propuestas y la generación automática de reportes de avance y evaluación. Se concluyó que la Unidad Didáctica Multimedia es un complemento didáctico al trabajo del docente de matemática, sobre todo, en la educación a distancia, ya que permitió la gestión autodidacta por parte del estudiante y logró su atención y su motivación.

Palabras clave: eXeLearning; Multimedia; Unidad didáctica; Estadística; Educación a distancia.

\section{Summary}

In Costa Rica, before 2016 the subject of statistics was not included in the national high school tests in Mathematics. As of 2016, the test, consisting of 60 items, includes 9 statistical items. On the other hand, teachers in their lessons usually use traditional expository methods, mediated by the use of a blackboard and printed materials. In this context, a need to create and implement new teaching techniques in this area was evident, for which a Multimedia Instructional Unit in Statistics was developed and applied through the eXeLearning technological tool. The instructional unit was applied to a group of 30 students belonging to the high school diploma for adults program from the region of Turrialba in Costa Rica. We used a qualitative, interpretive and naturalistic method based on the generation of analysis categories. It was determined that a simple way to show the information and the inclusion of review activities, exemplification, practice and evaluation activities, is desired and well valued by the students. In addition, it was observed that the technological means promoted student interest and motivation, while facilitating the monitoring of the proposed activities and the automatic generation of progress and evaluation reports. It was concluded that the Multimedia Instructional Unit is a didactic complement to the work of the teacher of mathematics, especially in distance education since it allowed self-taught management of the student and achieved their attention and motivation.

Keywords: eXeLearning; Multimedia; Instructional; Statistics; Distance Education.

\section{Introducción}

En Costa Rica, el Ministerio de Educación Pública (MEP) es el ente rector de la educación pública y privada. Dentro de las modalidades formativas ofertadas, se encuentra el programa de educación abierta. Según MEP (2016), la educación abierta "constituye una alternativa educativa donde el postulante escoge la manera en que se prepara según su disponibilidad de tiempo y avanza en sus estudios de acuerdo con sus posibilidades" (p.1). Además, esta modalidad educativa incluye el programa Bachillerato por Madurez, el cual está dirigido a personas mayores de dieciocho años que deseen concluir sus estudios de secundaria mediante la aprobación de las pruebas nacionales de bachillerato por madurez de diversas asignaturas. 
El Bachillerato por Madurez ofrece oportunidades de formación, pero el educando debe realizar un gran esfuerzo, ya que es necesario organizar y distribuir el tiempo para cumplir con sus diferentes responsabilidades y asumir un proceso de autoaprendizaje. Usualmente, dedicarse con exclusividad al estudio y contar con el apoyo de un maestro no es factible, por lo que es posible que el estudiante requiera de un material didáctico que le oriente en el proceso de aprendizaje, y a su vez, que despierte su motivación por aprender. El Estado de la Educación (2015), indica que el "cuido de menores, labores domésticas y obligaciones laborales afectan asistencia y permanencia de estudiantes" (p.249) mayores de edad en los sistemas educativos formales y que al desertar quedan desprovistos de medios adecuados para continuar autónomamente con sus estudios, siendo una necesidad contar con material didáctico que facilite la gestión de su propio aprendizaje.

Por otra parte, a pesar de que el MEP (2012) afirma que en la actualidad se requieren personas que sean "capaces de comprender, interpretar y usar la información para entender la realidad, resolver distintos problemas y tomar decisiones inteligentes" (p.55), y otorga reconocimiento a la matemática y a la estadística como parte de tal requerimiento de formación, antes del 2016 no se incluía estadística en las pruebas nacionales de bachillerato en la asignatura de Matemática. Así, a partir de 2016, la prueba, compuesta por 60 ítems, incluye 9 sobre estadística, es decir, se pasó nula presencia de la estadística en la prueba, a representar un $15 \%$ del total.

En una entrevista realizada por Ruiz (2016) a Lilliam Mora, directora de Gestión y Evaluación de la Calidad del MEP, se confirmó que la promoción en la prueba nacional de bachillerato por madurez en la asignatura de Matemática realizada en abril del 2016 fue de un $31,96 \%$. Mora explicó que en los últimos 15 años el porcentaje de personas que gana la prueba de bachillerato por madurez de Matemática en Costa Rica está entre 28\% y 35\%. Los estudiantes del sistema de Bachillerato por Madurez aprueban si obtienen una calificación en la prueba superior a 70, pero, según Mora, es común que el MEP aplique un intervalo de confianza de 10 puntos, y que los estudiantes aprueben con una nota de al menos 60 .

La problemática de las condiciones de independencia de los estudiantes en el sistema de Bachillerato por Madurez, incluyendo la falta de guía formal para su aprendizaje, además de la inclusión reciente de la estadística en las pruebas nacionales de bachillerato en la asignatura de Matemática y la baja tasa de aprobación, sugiere la necesidad de considerar la forma en que estos estudiantes se están preparando y justifica el interés por diseñar, aplicar y valorar una Unidad Didáctica Multimedia, con el propósito de crear un recurso que le facilite el autoaprendizaje de la estadística por parte de estos estudiantes.

Alfaro, Alpízar y Chávez (2012), señalaron que, en Costa Rica, para la enseñanza de la estadística y la probabilidad, los maestros suelen utilizar métodos expositivos tradicionales, mediados con pizarra y materiales impresos, con baja participación estudiantil y a causa de falta de recursos económicos para el acceso a laboratorios u otros recursos.

Ferro, Martínez y Otero (2009) indican que el uso de las Tecnologías de Información y Comunicación (TIC) en la enseñanza y aprendizaje, motiva, capta la atención, incita la actividad y el pensamiento, promoviendo la dedicación de más tiempo a trabajar y aprender mediante la interacción estudiante-ordenador.

Precisamente, una de las herramientas tecnológicas de desarrollo reciente para realizar mediación pedagógica es el eXeLearning. Según Saavedra (2011) "es un programa de edición de sitios web educativos de código abierto único por sencillez de su manejo y por las herramientas que incorpora" (p.24). El eXeLearning permite la edición de páginas con contenidos multimedia tales como; imágenes, vídeo, audio, animaciones, expresiones matemáticas, entre otras. Además, en varios estudios se ha determinado que esta herramienta: (a) facilita y mejora el aprendizaje, tanto teórico como práctico, aportando un cambio al esquema tradicional de la enseñanza 
haciendo que sea el estudiantado el principal actor del proceso de formación, otorgándole autonomía y oportunidad de desarrollar su creatividad, su capacidad de organización (Chao, 2014; Ulloque, 2016; Henoa \& Gonzales, 2014); y (b) reduce costos debido a su carácter de software libre, al tiempo que sus múltiples funcionalidades permiten fácil uso, visualización atractiva, integración de múltiples recursos, modificación y actualización (Mora, 2010).

Así, debido al interés de plantear una propuesta didáctica que permitiera dar solución, mediante un proceso de investigación, a la problemática identificada, se tomaron en cuenta las cualidades del eXeLearning para la gestión del autoaprendizaje y se desarrolló un estudio con el objetivo de diseñar una Unidad Didáctica Multimedia para realizar la mediación pedagógica con contenidos de estadística, tales como medidas de posición, medidas de variabilidad y representaciones para el análisis de información estadística. Unidad Didáctica Multimedia que facilitara el autoaprendizaje, que despertara el interés de los estudiantes por el estudio de la estadística y que a su vez permitiera la administración del tiempo según las necesidades específicas de cada estudiante.

Además, se valoró el diseño mediante su implementación con un grupo de 30 estudiantes de la región de Turrialba en Costa Rica, elegidos por conveniencia, matriculados en el programa de Bachillerato por Madurez durante la segunda convocatoria del año 2017, que no contaban con el tiempo necesario para optar por otros programas educativos presenciales y que tenían conocimientos básicos de computación y acceso a internet. Por medio de entrevista y observación se analizaron las acciones y percepciones de los estudiantes en una sesión de estudio utilizando la Unidad Didáctica Multimedia construida.

Según Rodríguez y Chacón (2008), un material multimedia se compone de variadas formas de presentar y procesar información (texto, imágenes, video, audio e interactividad), y según Marques (2011) un material multimedia educativo, utiliza todo ello con una finalidad de enseñanza y de aprendizaje. Para el desarrollo de un material multimedia, Belloch (2000), indica que se requiere de una serie de fases, a saber: "Análisis, Diseño del programa, Desarrollo del programa, Experimentación y validación del programa, Realización de la versión definitiva del programa, Elaboración del material complementario" (pp. 5-6).

Por ello, el proceso de diseño de la Unidad Didáctica Multimedia usando eXeLearning se realizó en cinco etapas o fases. La primera, la fase diagnóstica, en la que se determinaron características que debía tener la Unidad Didáctica Multimedia, mediante la consulta de referentes teóricos y de antecedentes, la opinión de expertos y de estudiantes de Bachillerato por Madurez. En la segunda fase se construyó una Unidad Didáctica Multimedia que tomara en consideración los resultados de la fase anterior. La tercera fase constituyó la validación de la propuesta, sustentándola en el juicio de expertos en didáctica de la matemática con amplia experiencia en enseñanza. Durante la cuarta etapa, se implementó la propuesta didáctica con estudiantes de Bachillerato por Madurez, con el propósito de realizar valoraciones y realizar acciones de mejora. En la quinta fase, se realizó una revisión y una adaptación de la propuesta inicial, tomando en cuenta las sugerencias y observaciones tanto de expertos como del estudiantado.

Todo el proceso se realizó bajo la consideración de aspectos del Análisis Didáctico de Rico (2016), como marco teórico general, y de aportes específicos de Saavedra (2011), Rodríguez y Chacón (2008), y Batanero, Godino, Green, Holmes y Vallecillos (2009).

Sobre las características que debería poseer un material multimedia, sobre todo, uno para educación a distancia, en la que se da asincronía entre emisor y receptor, se señalan: (a) estar al alcance de los estudiantes; (b) organizar el contenido por bloques, permitiendo lectura no lineal, interactividad, y libertad, pero garantizando la consecución global de los objetivos de aprendizaje; (c) contener actividades de adquisición, aplicación y evaluación del conocimiento; (d) aprovecha ventajas flexibilidad, inclusión y accesibilidad, y considera desventajas como costo, conectividad y capacitación (Saavedra, 2011). Además, Rodríguez y Chacón (2008) señalan los principios de 
coherencia, redundancia y señalización, de manera que: (a) se evita la sobrecarga sensorial y la repetición del contenido; (b) se resalta, de alguna manera, lo esencial.

Sobre planificación, implementación y valoración de unidades didácticas, en general, y como actividad principal del profesor, según Rico (2016) el análisis didáctico es una herramienta que permite esto, mediante: (a) articulación de los organizadores del currículo: contenidos, objetivos, metodología y evaluación; y (b) consideración de análisis del contenido (selección y organización), cognitivo (expectativas y actividades), de instrucción (materiales y recursos) y de evaluación (logro de expectativas).

Propiamente sobre la enseñanza y el aprendizaje de la estadística, Batanero, Godino, Green, Holmes y Vallecillos (2009), señalan errores y dificultades comunes que los estudiantes pueden presentar y que el profesor debe prever durante la planificación, entre ellos: (a) extrapolar contextos de aplicación; (b) lectura superficial de datos; (c) elección inadecuada de modo de representación; y (d) interpretación incompleta de media, mediana y desviación típica.

\section{Método}

Se siguió un método cualitativo naturalista interpretativo fundamentado en la generación de categorías de análisis, deductivamente según el marco teórico del análisis didáctico y otros referentes, e inductivamente según los propios hallazgos. Esto permitió identificar características que debía poseer dicha Unidad Didáctica Multimedia, generar una propuesta preliminar, validarla mediante juicio de expertos, y después de implementarla con un grupo de estudiantes, valorarla contemplando sus opiniones y mejorarla según la información recolectada mediante entrevistas semiestructuradas, observación y grupo focal. Precisamente, esto correspondió con las cinco fases del método, ya señaladas, y con los objetivos del estudio.

\section{Categorías de Análisis.}

Con el objetivo de generación de la propuesta preliminar y de interpretar los resultados de su implementación y valoración, se elaboraron 4 categorías de análisis basadas en Análisis Didáctico de Rico (2016) y en aportes de Saavedra (2011), Rodríguez y Chacón (2008), Batanero, Godino, Green, Holmes y Vallecillos (2009) y otros.

\section{Categoría 1: Planificación por parte del profesor.}

Esta categoría se define como el planeamiento didáctico que debe realizar el profesor. En este sentido, Picado (2006) lo concibe como una "tarea del docente para orientar la mediación entre el currículum establecido en los programas de estudio y el alumno. En esa tarea organiza y estructura los elementos del planeamiento didáctico (objetivos, contenidos, estrategias, etc.) para lograr un verdadero aprendizaje" (p.217).

Como subcategorías de análisis se tomaron en cuenta los cuatro componentes que propone Rico (2016): el análisis de contenido, el análisis cognitivo, el análisis de instrucción y el análisis de actuación.

Respecto al contenido, se incluyen las unidades de significado que se refieran a identificación, selección y organización de los conceptos y procedimientos utilizados en el desarrollo de la Unidad Didáctica Multimedia. En cuanto al análisis cognitivo, se incluyen las unidades de significado que demuestren que existen problemas de aprendizaje del tema de estadística por parte de los estudiantes. Por otro lado, en análisis de instrucción, se analiza la forma en que se selecciona, diseña y se da secuencia a las actividades utilizadas en la Unidad Didáctica, tomando en cuenta materiales y recursos utilizados para la mediación pedagógica. Por último, en análisis de actuación, se analiza si las actividades y herramientas de evaluación han 
logrado las expectativas de aprendizaje, de tal forma que cumplan con su funcionalidad eficazmente.

\section{Categoría 2: Inclusión de elementos de una Unidad Didáctica Interactiva.}

Esta categoría contempla la inclusión de elementos de una Unidad Didáctica. Al respecto, Corrales (2009) indica que

es importante considerar que los aprendizajes necesitan ser programados, en el sentido de que para abordarlos es preciso marcarse objetivos y contenidos, diseñar actividades de desarrollo y evaluación y prever los recursos necesarios. Las unidades didácticas, cualquiera que sea la organización que adopten, se configuran en torno a una serie de elementos que las definen (Corrales, 2009, p.7).

Se tomaron como subcategoría de análisis los elementos propuestos por Saavedra (2011), (a) herramientas que faciliten la adquisición de nuevos conocimientos, (b) actividades que permitan la aplicación de lo aprendido mediante experiencias significativas y (c) pruebas de evaluación que permitan la verificación de los conocimientos adquiridos.

\section{Categoría 3: Cumplimiento de los principios a seguir en la construcción de un multimedia.}

Esta categoría de análisis se refiere al cumplimiento de las normas o ideas fundamentales que se deben seguir para la elaboración de una Unidad Didáctica Multimedia.

Se tomaron como subcategorías de análisis los principios propuestos por Rodríguez y Chacón (2008), (a) principio de coherencia, (b) principio de redundancia y (c) principio de señalización. En el principio de coherencia se analiza el uso de sonidos e imágenes utilizados en la Unidad Didáctica desarrollada, según la opinión de los estudiantes. Sobre el principio de redundancia se analiza la impresión de los estudiantes con respecto a la presentación de la información en relación la presencia de redundancia. Y en la señalización se analiza la percepción de los estudiantes en relación al aspecto y forma de presentación de los contenidos multimedios de la Unidad Didáctica.

\section{Categoría 4: Descripciones de las fortalezas, limitaciones, errores y dificultades presentadas por los estudiantes.}

En esta categoría de análisis se analizan fortalezas, limitaciones, errores y dificultades de los estudiantes durante la aplicación de la Unidad Didáctica.

Saldaña, Escartín, Peña, Jiménez, Ceja, Rey, Vidal y Rodríguez (2014) definen las fortalezas como "formas de comportamiento, pensamientos y emociones que se asocian con un rendimiento óptimo" (p.1). Como subcategoría de análisis se plantaron las fortalezas que se considera que la Unidad Didáctica Multimedia permite desarrollar en los estudiantes. Según Argüís, Pilar, Bolsas, Hernández y Salvador (2012) entre las principales fortalezas se encuentran: la Curiosidad, la apertura mental, el amor por el aprendizaje, la perseverancia, la vialidad y la esperanza. En la tabla 1 se muestran los conceptos de cada una de las fortalezas. 
Tabla 1.

Concepto de las principales fortalezas que pueden ser desarrolladas gracias al uso de la Unidad Didáctica Multimedia.

\begin{tabular}{|c|c|}
\hline Fortaleza & Concepto \\
\hline $\begin{array}{l}\text { Curiosidad (interés por el mundo, } \\
\text { búsqueda de novedades, apertura a } \\
\text { experiencias). }\end{array}$ & $\begin{array}{l}\text { Tener interés por lo que sucede en el mundo, } \\
\text { encontrar temas fascinantes, explorar y } \\
\text { descubrir nuevas cosas. }\end{array}$ \\
\hline $\begin{array}{l}\text { Apertura mental (juicio, pensamiento } \\
\text { crítico). }\end{array}$ & $\begin{array}{l}\text { Pensar sobre las cosas y examinar todos sus } \\
\text { significados y matices. No precipitarse al sacar } \\
\text { conclusiones, sino tras evaluar cada posibilidad. } \\
\text { Ser capaz de cambiar las propias ideas en base } \\
\text { a la evidencia. Ponderar adecuadamente cada } \\
\text { evidencia. }\end{array}$ \\
\hline Amor por el aprendizaje. & $\begin{array}{l}\text { Llegar a dominar nuevas habilidades, materias y } \\
\text { conocimientos, ya sea por sí mismo o mediante } \\
\text { un aprendizaje formal. Está obviamente } \\
\text { relacionada con la fortaleza de curiosidad pero } \\
\text { va más allá, describiendo una tendencia } \\
\text { sistemática a añadir cosas a lo que uno sabe. }\end{array}$ \\
\hline $\begin{array}{l}\text { Perseverancia (tenacidad, diligencia, } \\
\text { laboriosidad). }\end{array}$ & $\begin{array}{l}\text { Terminar lo que uno empieza. Persistir en una } \\
\text { actividad aunque existan obstáculos. Obtener } \\
\text { satisfacción por las tareas emprendidas y que } \\
\text { consiguen finalizarse con éxito. }\end{array}$ \\
\hline $\begin{array}{l}\text { Vitalidad (ánimo, entusiasmo, vigor, } \\
\text { energía). }\end{array}$ & $\begin{array}{l}\text { Afrontar la vida con entusiasmo y energía; hacer las } \\
\text { cosas con convicción y dando todo de uno } \\
\text { mismo; vivir la vida como una apasionante } \\
\text { aventura; sentirse vivo y activo. }\end{array}$ \\
\hline $\begin{array}{l}\text { Esperanza (optimismo, proyección hacia } \\
\text { el futuro). }\end{array}$ & $\begin{array}{l}\text { Esperar lo mejor para el futuro y trabajar para } \\
\text { conseguirlo; creer que un buen futuro es algo } \\
\text { que está en nuestras manos conseguir. }\end{array}$ \\
\hline
\end{tabular}

Fuente: Elaboración propia. Basado en Argüís, Pilar, Bolsas, Hernández y Salvador (2012).

Por otro lado, Godino, Batanero y Font (2003) definen que se comete un error "cuando el alumno realiza una práctica (acción, argumentación, etc.) que no es válida desde el punto de vista de la institución matemática escolar" (p.69). Así mismo, Godino et al. (2003) definen dificultad como "el mayor o menor grado de éxito de los alumnos ante una tarea o tema de estudio" (p.69).

\section{Muestra.}

Para valorar el uso de los estudiantes de la Unidad Didáctica Multimedia, se eligió, por conveniencia, una muestra de 30 estudiantes (6 hombres y 24 mujeres) del programa de Bachillerato por Madurez de la región de Turrialba en Costa Rica, durante la segunda convocatoria del 2017. Las edades oscilaron entre 18 y 44 años, con una moda de 24 años (para un 16,66 por ciento) y un promedio de 27.83 años.

\section{Recolección y sistematización de datos.}

Para valorar la Unidad Didáctica Multimedia se aplicaron tres técnicas de recolección de datos: entrevista semiestructurada, observación y grupo focal.

Con el fin de realizar un diagnóstico de necesidades, que permitió obtener información relevante a tomar en cuenta en el diseño y desarrollo de la Unidad Didáctica Multimedia, se aplicó una entrevista semiestructurada a los 30 estudiantes de la muestra. Se utilizaron distintos tipos de preguntas (generales, de opinión, de contraste y de expresión de sentimientos), pero de manera que se admitía el surgimiento de nuevas preguntas y cuestiones. 
También, se realizaron observaciones en el ambiente natural de los estudiantes y durante el desarrollo de una sesión de estudio usando la Unidad Didáctica Multimedia. Se observó interés del estudiante por el uso de la herramienta, motivación, características ambientales, tiempo que dedica al uso de la herramienta, utilización efectiva del recurso, interrupciones, entre otros aspectos.

Además, se realizó una entrevista tipo grupo focal con el fin de recolectar impresiones referentes al uso de la Unidad Didáctica Multimedia, para posteriormente analizar la información obtenida y realizar mejoras a la misma. El grupo focal se aplicó a un grupo de 10 estudiantes de la muestra elegidos al azar. Para la recolección y posterior sistematización de la información, se utilizó una guía para grupo focal. En este sentido, el investigador guio las preguntas pertinentes al tema de estudio, a la vez que se encargó de la observación y anotaciones respectivas.

La información recolectada fue sistematizada mediante matriz en hoja de cálculo de Microsoft Office Excel, organizada según las categorías de análisis previamente establecidas (deductivamente), teniendo en cuenta que en esta etapa podría, eventualmente, surgir alguna otra (inductivamente) Finalmente, las categorías codificadas se agruparon en temas y patrones.

\section{Triangulación.}

Hernández, Fernández y Baptista (2014), define triangulación en investigación como la "utilización de diferentes fuentes y métodos de recolección." (p.418), agrega que, "en la indagación cualitativa poseemos una mayor riqueza, amplitud y profundidad en los datos, si éstos provienen de diferentes actores del proceso, de distintas fuentes y al utilizar una mayor variedad de formas de recolección de los datos" (p.439) y, además, apoya la validez de los resultados obtenidos de la información recolectada.

Precisamente, la entrevista semiestructurada, la observación y el grupo focal, permitieron obtener datos de diversas formas y en diferentes momentos, y organizar e interpretar la información para el logro de los objetivos del estudio.

La entrevista permitió identificar características que debía poseer la Unidad Didáctica Multimedia en contraste de los lineamientos teóricos identificados previamente. Las observaciones no participativas, en el ambiente natural o escenario que el estudiante utiliza en su vida cotidiana y durante una sesión de estudio con la Unidad Didáctica Multimedia, permitió determinar acciones, percepciones, estados de ánimo, actitudes, conflictos, y demás aspectos, en contraste de lo planificado durante el diseño. Y el grupo focal, permitió valorar el proceso de implementación de manera global, identificar opiniones, recomendaciones y consideraciones que deberían ser incluidas en una nueva etapa de rediseño.

\section{Resultados}

De acuerdo con los objetivos de este estudio, los resultados abarcan: (a) una propuesta de Unidad Didáctica Multimedia, un diseño inicial según la identificación previa de características que debía poseer, y un rediseño a partir de los resultados de la implementación; y (b) una valoración de la Unidad Didáctica Multimedia, de diseño e implementación, mediante el contraste de las opiniones estudiantiles y los referentes teóricos y antecedentes contemplados.

Los resultados sugieren que el diseño y la aplicación de una Unidad Didáctica Multimedia, como recurso para estudiantes del sistema de Bachillerato por Madurez, que enfrentan condiciones de independencia, falta de guía para su aprendizaje, y dificultades específicas para el aprendizaje de la estadística, no solo facilita el autoaprendizaje, si no que motiva y entretiene. Sin embargo, los resultados también sugieren que las cualidades del recurso 
dependen en gran medida de su planificación y de su diseño, por parte del profesor y en consideración de múltiples aspectos teórico, prácticos y experimentales, tal y como lo sugirió, Belloch (2000), en las fases para el desarrollo de un material multimedia.

\section{Descripción de la Unidad Didáctica Multimedia diseñada.}

Sobre organizar el contenido por bloques, permitiendo lectura no lineal, interactividad, y libertad, pero garantizando la consecución global de los objetivos de aprendizaje, sobre contener actividades de adquisición, aplicación y evaluación del conocimiento (Saavedra, 2011), y sobre la articulación de los organizadores del currículo: contenidos, objetivos, metodología y evaluación (Rico, 2016), la Unidad Didáctica Multimedia diseñada posee un menú principal que incluye ocho secciones: Presentación, Conocimiento Previo, Capítulo 1, Capítulo 2, Capítulo 3, Capítulo 4, Evaluación Final y Referencias Bibliográficas. El menú principal se puede observar en la figura 1.

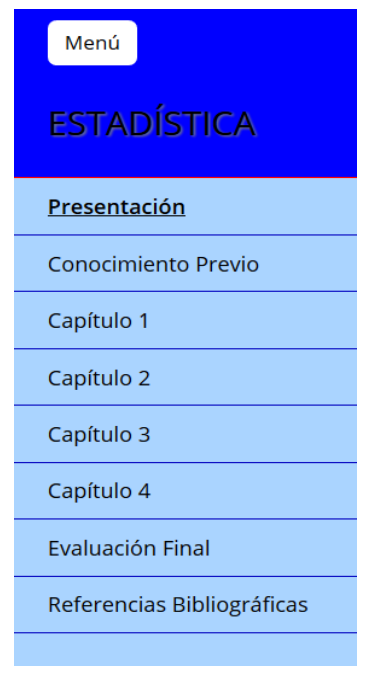

Figura 1. Menú principal de la Unidad Didáctica Multimedia

Específicamente sobre accesibilidad y capacitación (Saavedra, 2011), en la sección de presentación se incluyen aspectos generales que el estudiante debe considerar para poder utilizar de forma adecuada la Unidad Didáctica Multimedia.

Específicamente sobre análisis del contenido, su selección y organización (Rico, 2016), en la sección de Conocimiento Previo el estudiante tiene la posibilidad de estudiar los conceptos estadísticos básicos que debe conocer antes de iniciar el estudio de los cuatro capítulos indicados.

Específicamente sobre análisis cognitivo, expectativas y actividades (Rico, 2016), cada uno de los cuatro capítulos corresponde a una habilidad general establecida por el MEP (2017), para el área temática de Estadística del nivel educativo:

Habilidad 1. Valorar la importancia de las medidas de resumen (posición) para el análisis de la información estadística. Además de utilizar las medidas de posición para resumir y analizar la información proveniente de un grupo de datos cuantitativos.

Habilidad 2. Valorar la importancia de las medidas de resumen (variabilidad) para el análisis de la información estadística además de utilizar las principales medidas de variabilidad para evaluar y comparar la dispersión de los datos.

Habilidad 3. Utilizar diferentes representaciones para analizar la posición y variabilidad de un conjunto de datos.

Habilidad 4. Analizar la importancia del uso de medidas relativas de tendencia central y variabilidad dentro de los análisis comparativos de información. 
Cada capítulo se aborda bajo el enfoque de resolución de problemas, de manera que el estudiante puede abarcar los contenidos y realizar las actividades sin participación de algún profesor. Además, sobre la articulación de los organizadores del currículo: contenidos, objetivos, metodología y evaluación (Rico, 2016), cada capítulo se subdivide en cuatro secciones:

Conceptos. Se incluyen los fundamentos teóricos de la temática de estadística tratada, mediante la técnica de resolución de problemas. La sección se inicia brindando una situación problema en la que se expone una serie de interrogantes que surgen de la misma. Posteriormente, se brinda la solución a las interrogantes, a la vez que se introducen los conceptos estadísticos referentes a la situación planteada.

Práctica. Se brinda la posibilidad de resolver ejercicios y problemas guiados, de tal forma que el estudiante tiene acceso a pistas y sugerencias para una mejor comprensión de los conceptos estadísticos estudiados en el capítulo.

Ejercicios de Evaluación. Se presentan ejercicios sin sugerencias. Además, se muestra una calificación al terminar la actividad.

Solución a los ejercicios de Evaluación. Se incluye, mediante un documento en formato PDF, la solución detallada de los ejercicios de evaluación.

En la figura 2 se muestra una subsección del capítulo 1.

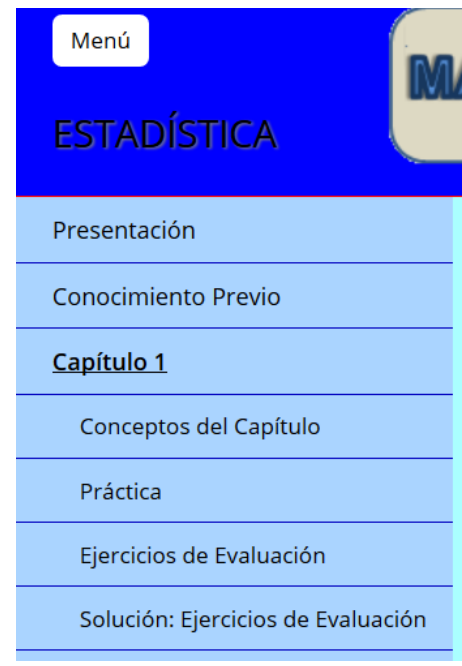

Figura 2. Subsecciones capítulo 1 de la Unidad Didáctica Multimedia

Específicamente sobre análisis de evaluación y logro de expectativas (Rico, 2016), en la sección de Evaluación Final, se incluyen ejercicios y problemas referentes a todos los temas de estadística evaluados en la prueba de Bachillerato por Madurez durante el periodo 2017. La Evaluación Final está compuesta por nueve ítems distribuidos en las cuatro habilidades generales tratadas en los cuatro capítulos. Para la resolución de la evaluación final el estudiante cuenta con un límite de tiempo de tres minutos por ítem (27 minutos, en total).

\section{Valoración de diseño e implementación de la Unidad Didáctica Multimedia.}

El diseño y la implementación de la Unidad Didáctica Multimedia fueron valorados a partir de la información recolectada de entrevistas, observaciones y grupo focal con los estudiantes y en términos de las categorías de análisis establecidas.

\section{Planificación por parte del profesor.}

La planificación por parte del profesor contempla 4 componentes, según Rico (2016): el análisis de contenido, el análisis cognitivo, el análisis de instrucción y el análisis de evaluación. 
Respecto al análisis de contenido, fueron las habilidades pretendidas, según el temario de evaluación de las Pruebas de Bachillerato por Madurez de Matemáticas aplicadas en la segunda convocatoria del año 2017, las que guiaron la estructura de los contenidos y de los conceptos afines incluirlos en la Unidad Didáctica Multimedia.

En cuanto al análisis cognitivo, mediante el análisis de referentes teóricos, antecedentes, entrevistas y observaciones, además de la identificación de las expectativas de aprendizaje, se determinaron posibles problemas en el estudio de la estadística. Se observó que la mayoría de los estudiantes desconocían conceptos básicos de estadística, por lo que se decidió incluir la sección de conocimientos previos y su respectivo instrumento de evaluación. Además, se consideró que la Unidad Didáctica Multimedia debía contener explicaciones claras y concretas para el abordaje de los nuevos contenidos y el desarrollo de las habilidades. Así, durante la implementación, no se observaron problemas para la comprensión y la aplicación de los conceptos estadísticos abordados. Inclusive, los estudiantes indicaron que, durante el desarrollo de las actividades propuestas, comprendían y se concentraban, desconectándose del resto del mundo, olvidando las preocupaciones que los aqueja, y calificando de "adictivo" el hecho de que una vez iniciada una actividad no se querían detener hasta concluirla.

Al respecto del análisis de instrucción, los estudiantes indicaron que la organización del contenido de la Unidad Didáctica Multimedia permitió una interacción atractiva, facilitó el acceso a los recursos, y a las actividades de práctica y de auto evaluación.

Sobre análisis de evaluación, en primer lugar, en las actividades de práctica, se observó que las mismas favorecieron el aprendizaje de la materia. Respecto al principio señalización indicado por Rodríguez y Chacón (2008), y a las posibles dificultades de aprendizaje de conceptos estadísticos señaladas por Batanero, Godino, Green, Holmes y Vallecillos (2009), se incluyeron sugerencias que guiaban el estudio de conceptos, de tal forma que, si en algún momento surgía alguna duda en la resolución de uno de los problemas, el estudiante podía consultar la sugerencia y basarse en ella para aclararse y continuar. En segundo lugar, respecto a las actividades de evaluación parcial y final, se observó motivación por parte de los estudiantes, al observar sus calificaciones, automáticamente generadas y como reflejo de lo que habían aprendido. Además, se incluyó una selección de ejercicios similares a los aplicados en las pruebas nacionales de bachillerato por madurez, con el fin de que el estudiante analizara y resolviera problemas y ejercicios similares a los que enfrentaría en el desarrollo de la prueba oficial.

Se utilizó el software HotPotatoes para desarrollar las actividades de práctica y de evaluación, pues ofrece una calificación, con la que el estudiante puede medir lo que ha aprendido durante su sesión de estudio. En este sentido, y según lo señalado por Ferro, Martínez y Otero (2009) sobre promoción de la motivación, la atención, la actividad y el pensamiento, promoviendo la dedicación de más tiempo a trabajar y a aprender mediante la interacción estudiante-ordenador, los estudiantes indicaron excelente que el programa brinde la respuesta inmediatamente después de responder a la pregunta y no hasta concluida la prueba, ya que esto les permite analizar el por qué la respuesta es incorrecta y proceder a corregirla. Así mismo, indicaron que el tiempo se va muy rápido al usar la Unidad Didáctica Multimedia, que utilizarían la herramienta en cualquier momento que les quedara un tiempo libre. Además, agregaron que las actividades de evaluación dan la idea de cuánto se ha aprendido, lo que permite suponer que las actividades y herramientas de evaluación despiertan la motivación y el interés de los estudiantes. Se observó que un estudiante al obtener una nota inferior al 100, vuelve a resolver toda la actividad nuevamente con la intensión de alcanzarlo.

\section{Inclusión de los elementos de una Unidad Didáctica Interactiva.}

La inclusión de los elementos de una Unidad Didáctica Interactiva, según Saavedra (2011), contempla: (a) herramientas que faciliten la adquisición de nuevos conocimientos; (b) 
actividades que permitan la aplicación de lo aprendido mediante experiencias significativas; y (c) pruebas de evaluación que permita la verificación de los conocimientos adquiridos.

Respecto a las herramientas que faciliten la adquisición de nuevos conocimientos, los estudiantes indicaron que les pareció excelente la introducción de la materia por medio de una situación problema, ya que esto les permitió comprender la utilidad real que tiene en la vida cotidiana. Además, se incluyeron actividades en las que se presentara la información de diversas formas, por ejemplo, se propuso un asocie elaborado con el programa HotPotatoes, donde se debían relacionar los conceptos básicos de estadística con su respectivo significado (ver figura 3).

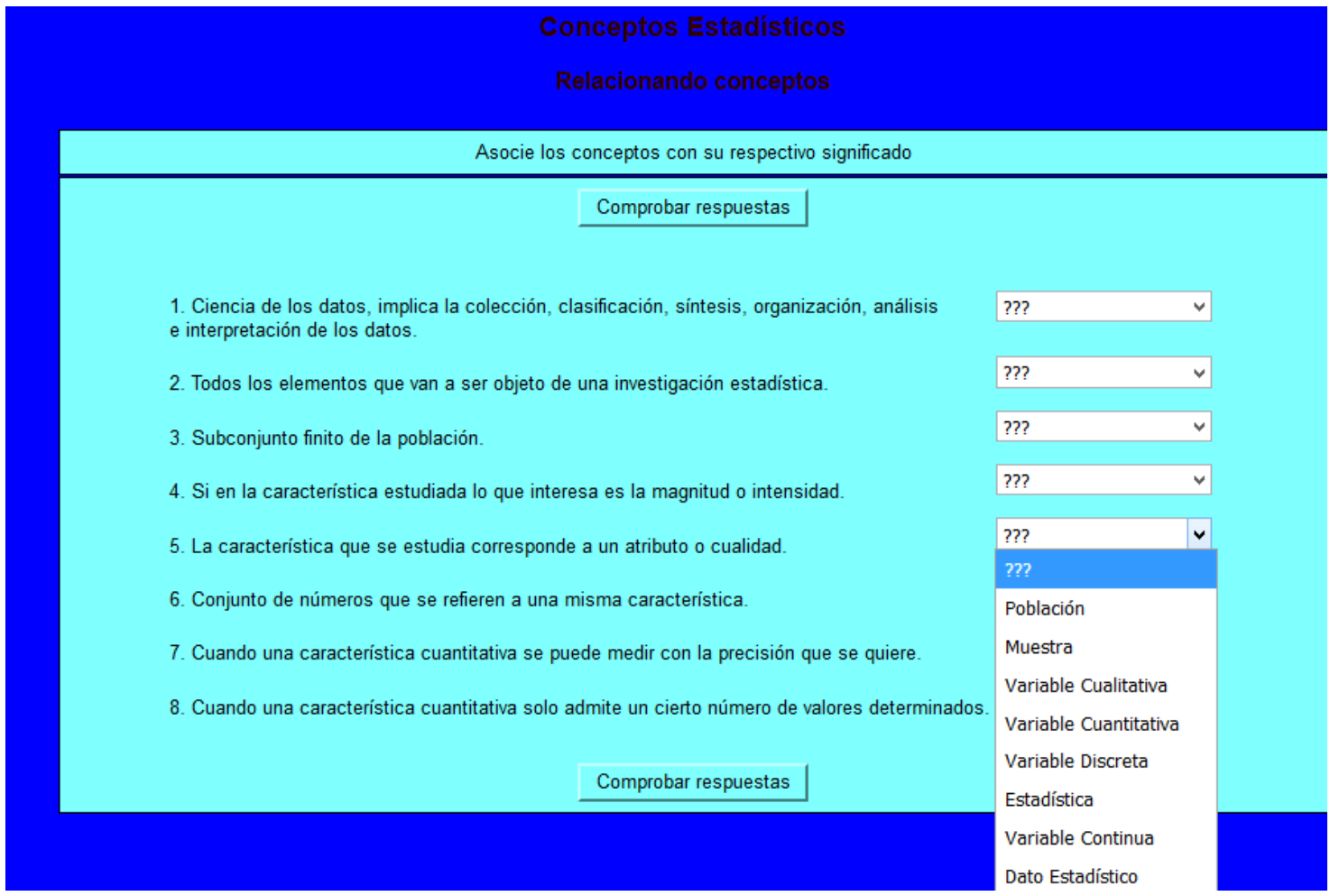

Figura 3. Asocie desarrollado con el software hotpotatoes

En cuanto a las actividades que permitan la aplicación de lo aprendido mediante experiencias significativas, se incluyeron secciones de Práctica, cuyos ejercicios fueron tomados de pruebas aplicadas anteriormente por el Ministerio de Educación Pública. Además, en cada uno, se incluyó una sugerencia para su solución, que podía ser consultada en caso de ser requerida. Al respecto, los estudiantes indicaron que estas sugerencias son una característica enriquecedora del material que facilita el aprendizaje de los conceptos.

Respecto a las pruebas de evaluación, se construyó una evaluación final para cada capítulo de la Unidad Didáctica Multimedia. Cada una de las actividades de evaluación fue elaborada con ejercicios tomados de exámenes de matemática aplicados anteriormente por el Ministerio de Educación Pública en las Pruebas de Bachillerato por Madurez. El tipo de evaluación aplicada, permite conocer de forma inmediata la calificación obtenida en cada pregunta que se resuelve, lo que posibilita que el estudiante determine si resolvió de forma correcta, el problema o ejercicio, o si por el contrario, debe repasar los conceptos necesarios para determinar una solución satisfactoria. Los estudiantes opinaron que la evaluación por medio de ítems similares a los aplicados en las pruebas de bachillerato permite un acercamiento a la realidad que enfrentarán y autoevaluarse en cuanto a los conocimientos previos adquiridos antes de avanzar con los demás contenidos de la Unidad Didáctica Multimedia. 
Tanto para las actividades de práctica como para las de evaluación, se construyeron ítems de selección única y de respuesta cerrada. La mayoría de los ítems propuestos fueron de selección única, pues favorecían la interacción entre estudiante y Unidad Didáctica Multimedia, y el reconocimiento del tipo de pregunta que se presenta en el examen de Matemática de Bachillerato por Madurez.

\section{Cumplimiento de los principios a seguir en la construcción de un multimedia.}

El cumplimiento de los tres principios a seguir en la construcción de una Unidad Didáctica Multimedia de Rodríguez y Chacón (2008), contempla: (a) el principio de coherencia, (b) el principio de redundancia, y (c) el principio de señalización.

En cuanto al principio de coherencia, se analizó el uso de sonidos e imágenes. De acuerdo con los resultados del análisis de literatura y del diagnóstico, se evitó el uso de imágenes ajenas a la presentación de los contenidos, de forma que las imágenes y cuadros que se presentaran serían las de vital importancia para la comprensión de los conceptos estadísticos. Según la opinión de los estudiantes, el uso del sonido en el video que se presentó en la sección de conocimiento previo no era necesario, ya que estos señalaron "que el sonido estaba de más", lo que concuerda con el principio de coherencia que establece Rodríguez y Chacón (2008).

Respecto al principio de redundancia, dados los resultados del análisis del diagnóstico de necesidades y de la revisión de la literatura, se evitó el uso de redundancia de cualquier tipo en el desarrollo de la Unidad Didáctica Multimedia. Los estudiantes indicaron que se utilizó un lenguaje sencillo, de fácil interpretación, sin saturación de información, que fue muy bien seleccionada y específica del tema.

En cuanto a la señalización, de acuerdo con las herramientas compatibles con el software eXeLearning, se creó un menú contextual al lado izquierdo de la pantalla, con el objetivo de guiar al estudiante en la navegación. Así mismo, se incluyó en cada sección del menú lateral, una sección de instrucciones generales, en la que se establecieron aspectos importantes que debía conocer el estudiante para poder desarrollar sin inconvenientes la sección respectiva. Los estudiantes aseguraron que la Unidad Didáctica Multimedia es muy versátil, pues permite la navegación por cada una de sus secciones de forma ágil y rápida.

\section{Descripciones de las fortalezas, limitaciones, errores y dificultades presentadas por los estudiantes.}

Para la descripción de fortalezas, limitaciones, errores y dificultades presentadas por los estudiantes, se tomaron en cuenta, tanto las que se identificaron en el aprendizaje de la estadística, como las que se presentaron en el uso de la plataforma interactiva desarrollada.

Respecto a las fortalezas presentadas por los estudiantes se identificaron curiosidad, apertura mental, amor por el aprendizaje, perseverancia, vitalidad y esperanza. En cuanto a curiosidad, el estudiante indicó que la Unidad Didáctica Multimedia permitió manipular los conceptos estadísticos de forma diferente en relación con otras técnicas de estudio. Sobre apertura mental, se observó que el estudiante analiza muy bien la pregunta antes de marcar la respuesta definitiva, esto con el fin de obtener una calificación satisfactoria en la actividad que está desarrollando. Así mismo, sobre amor por el aprendizaje, los estudiantes indicaron que la presentación de los contenidos y las actividades permitía la adquisición de conocimientos y habilidades de forma escalonada, y les resultaba necesario seguir avanzando y siguiendo el orden sugerido para poder obtener mejores resultados. En cuanto a perseverancia, se observó que los estudiantes que no habían podido obtener una nota superior a 70 en las actividades la resolvían de nuevo hasta conseguirlo. Por otro lado, en cuanto a vitalidad, aun con las diferentes responsabilidades de los estudiantes (trabajo, familia, estudio, entre otras), desarrollaron las 
actividades propuestas con entusiasmo y convicción de aprender. Por último, sobre esperanza, los estudiantes se mostraron optimistas con el avance en el desarrollo de las actividades.

Respecto a las dificultades del uso de la Unidad Didáctica Multimedia, una de las limitaciones presentada durante su aplicación, fue un problema de permisos de seguridad por parte del navegador en una de las computadoras, esto causó que no se desplegara la información de las actividades de evaluación desarrolladas con el software Hotpotatoes. Sin embargo, esta dificultad se solucionó, fácil y rápidamente, al brindar los permisos necesarios al navegador.

Por otro lado, respecto al aprendizaje de la estadística, algunos de los errores cometidos por los estudiantes se basaron en realizar mal los cálculos matemáticos. Por ejemplo, al calcular la media aritmética, los estudiantes ingresaban en la calculadora la suma de todos los datos y al final incluían la división entre la cantidad total de los mismos, por lo que la calculadora resolvía primero la división del último dato de la suma con el total de datos. Este error, no había sido contemplado entre los señalados por Batanero, Godino, Green, Holmes y Vallecillos (2009).

\section{Discusión}

El fin principal del trabajo fue elaborar y aplicar una Unidad Didáctica Multimedia sobre estadística, utilizando la herramienta tecnológica eXeLearning trabajando con un grupo de estudiantes de bachillerato por madurez de la región de Turrialba en Costa Rica. Esto se logró mediante la identificación de características que debía poseer dicha Unidad Didáctica Multimedia, la generación de una propuesta preliminar, su validación mediante juicio de expertos, y su implementación con un grupo de 30 estudiantes. La identificación de características deseables en el recurso se fundamentó en la revisión teórica y de antecedentes (Rodríguez y Chacón, 2008; Rico, 2016; Corrales, 2009), y se contrastó con un diagnóstico de necesidades y la opinión estudiantil.

Se encontró que la Unidad construída permitió mostrar de manera simple y práctica la información, esto al tomar en cuenta los principios de coherencia, redundancia y señalización (Rodríguez y Chacón, 2008), y la inclusión de actividades de repaso, ejemplificación, práctica y evaluación (Saavedra, 2011); todo ello favoreció el autoaprendizaje y fueron valoradas positivamente por los estudiantes.

También, se observó que el medio tecnológico promovió interés y motivación estudiantil, al tiempo que facilitó el seguimiento de las actividades propuestas y la generación automática de reportes de avance y evaluación. Esto coincidió con los trabajos realizados por Mora (2010) y Ferro, Martínez y Otero (2009).

Además, se encontró que la Unidad Didáctica Multimedia elaborada es un complemento didáctico al trabajo del docente de matemática con sus estudiantes, sobre todo en el caso de la Educación Abierta, ya que aunque requierió de planificación por parte del profesor, permite la gestión autodidacta del estudiante, lo cual cohincide con trabajos de Belloch (2000); Chao (2014); Ulloque (2016); y Henoa y Gonzales (2014). Los resultados sugieren que la utilidad del recurso depende de la planificación y diseño por parte del profesor, según sus intenciones e incluyendo las mejoras luego de cada implementación.

Por otra parte, como aportes novedosos de este estudio se encuentran que la Unidad Didáctica desarrollada: (a) facilitó el trabajo diario de los estudiantes, dado que les permitió dedicar tiempo a otras labores también importantes para ellos; (b) logró que los estudiantes de educación a distancia comprendieran mejor y más fácilmente la estadística; (c) permite que cada estudiante pueda planificar su tiempo y avanzar de acuerdo a su ritmo de aprendizaje, pues cada persona avanza independientemente del avance de las demás; (d) cuenta con todos los contenidos de estadística que se evalúan en la prueba nacional de bachillerato por madurez de Matemática, 
lo que permite que el estudiante no tenga que estar buscando información en otras fuentes; (e) evidencia fortalezas estudiantiles como curiosidad, apertura mental, amor por el aprendizaje, perseverancia, vitalidad y esperanza.

Otros aportes novedosos de este estudio son: (a) el software HotPotatoes, para la elaboración de instrumentos de evaluación, permite no solo captar la atención del estudiante y que valore el avance de su propio aprendizaje, sino que posee la ventaja de brindar una calificación inmediatamente después de responder cada pregunta lo que permite analizar la pregunta nuevamente y verificar el resultado; (b) la implementación de una sección donde se incluya el solucionario de las actividades de evaluación con el procedimiento detallado para dar solución a la situación planteada permite al estudiante comprender mejor el error cometido durante la actividad desarrollada; (c) es conveniente que los ejercicios se asemejen a los ítems de evaluación, con el fin de acercar lo más posible el estudiante al análisis de problemas y ejercicios similares a los que enfrentará en el desarrollo de la prueba final; (d) en las prácticas, es provechosa la inclusión de sugerencias y aclaraciones que guíen el estudio de los conceptos y que puedan ser consultadas en el momento que se requiera; (e) la sección de conocimientos previos permite que el estudiante tenga la opción de realizar consultas y asegurase de poder avanzar con nuevos temas; (f) el enfoque de resolución de problemas motiva al estudiante a resolver situaciones que se presentan en la vida cotidiana, y a su vez, sirven de preámbulo para abarcar los contenidos planteados.

En general, los resultados sugieren que la Unidad Didáctica Multimedia como recurso para estudiantes de un sistema de educación a distancia que enfrentan problemáticas debidas a la independencia, a la falta de guía y a dificultades específicas para el aprendizaje, facilitó el autoaprendizaje a la vez que logró la atención y motivación de los estudiantes.

\section{Referencias}

Alfaro, A., Alpízar, M., \& Chaves, E. (2012). Recursos metodológicos utilizados por docentes de I y II ciclos de la educación general básica en la dirección regional de Heredia, al impartir los temas de probabilidad y estadística. Uniciencia, 26, 135 - 151. Recuperado de: http://www.fcen.una.ac.cr/uniciencia/Vol_26_N1-2(Paper_13).pdf

Argüís, R., Bolsas, A., Hernández, S., \& Salvador M. (2012). Psicología Positiva aplicada a la Educación. Programa "Aulas Felices". Recuperado de: https://www.educacion.navarra.es/documents/27590/203401/Aulas+felices+documenta ci\%C3\%B3n.pdf/3980650d-c22a-48f8-89fc-095acd1faa1b

Batanero, C., Godino, J., Green, D., Holmes, P., \& Vallecillos, A. (2009). Errores y dificultades en la comprensión de los conceptos estadísticos elementales. Internation Journal of Mathematics Education in Science and Technology, 25(4), 527-547.

Belloch, M. (2000). Desarrollo de Aplicaciones Multimedia Interactivas. Unidad de Tecnología Educativa (UTE). Universidad de Valencia: España. Recuperado de: https://www.uv.es/bellochc/pdf/pwtic5.pdf

Chao, K. (2014). Estrategias didácticas mediadas con TIC en un curso de expresión oral francesa. Revista Actualidades Investigativas en Educación, 14(2), 01-30. Recuperado de: https://www.redalyc.org/pdf/447/44731371007.pdf

Corrales, A. (2009). La programación a medio plazo dentro del tercer nivel de concreción: Las Unidades Didácticas. Revista Digital de Educación Física, 1(2), 41-53. Recuperado de: https://emasf.webcindario.com/La_programacion_a_medio_plazo_dentro_del_tercer_ni ve_\%20de_concrecion_unidades_didacticas.pdf

Estado de la Educación (2015). V informe del estado de la educación. Costa Rica. Recuperado de: http://www.estadonacion.or.cr/educacion2015/assets/carpeta_iee_web.pdf 
Ferro, C., Martínez, A., \& Otero, M. (2009). Ventajas del uso de las TICs en el proceso de enseñanza-aprendizaje desde la óptica de los docentes universitarios españoles, Edutece, 29, Doi: https://doi.org/10.21556/edutec.2009.29.451

Godino, J., Batanero C., \& Font V. (2003). Fundamentos de la enseñanza y aprendizaje de la Matemática para maestros. Universidad de Granada. Recuperado de: https://www.ugr.es/ jgodino/edumat-maestros/manual/1_Fundamentos.pdf

Henoa, A., \& Gonzales, L. (2014). Elaboración de un ambiente virtual colaborativo usando eXeLearning para la enseñanza de Ciencias Naturales. Ventana Informática, 31(2), 115130. Doi: https://doi.org/10.30554/ventanainform.31.520.2014

Hernández, R., Fernández, C. y Baptista, M. (2014). Metodología de la Investigación. Sexta Edición. México D.F.: Mac Graw Hill

Marqués, P. (2011). Multimedia Educativo: Clasificación, Funciones, Ventajas, Diseño de Actividades. Departamento de Pedagogía Aplicada, Facultad de Educación, UAB. Recuperado de: https://posgradouat.files.wordpress.com/2011/05/multimediaeducativo.pdf

Ministerio de Educación Pública (MEP, 2012). Programas de Estudio Matemática. Recuperado de: http://www.mep.go.cr/sites/default/files/programadeestudio/programas/matematica.pdf

Ministerio de Educación Pública (MEP, 2016). ABC de los Programas de Educación Abierta. Disponible en: http://www.mep.go.cr/sites/default/files/blog/ajduntos/abc-programaseducacion-abierta-dgec.pdf

Ministerio de Educación Pública (MEP, 2017). Temario prueba de Matemática Bachillerato por Madurez Suficiente.

Mora, F. (2010). Uso del software libre para el contexto educativo a distancia: Experiencia con el uso del exe-learnig y el moodle para la implementación de cursos virtuales. Revista Calidad en la Educación Superior, 1(2), 136-149. Recuperado de: https://investiga.uned.ac.cr/revistas/index.php/revistacalidad/article/view/412/307

Picado, F. (2006). Didáctica General: Una Perspectiva Integradora. Costa Rica: .Universidad Estatal a Distancia.

Rico, L. (2016). Matemáticas y análisis didáctico. En L. Rico y A. Romero (Eds.), Elementos de didáctica de la matemática para el profesor de secundaria (pp. 85-100). Madrid: Ediciones Pirámide.

Rodríguez, S., \& Chacón, M (2008). Bases Teóricas y Consideraciones Prácticas en la Elaboración de Material Multimedia Para un Curso de Cálculo. Revista Electrónica Actualidades Investigativas en Educación, 8(1), 01-30. Recuperado de: https://revistas.ucr.ac.cr/index.php/aie/article/view/9312/17780

Ruiz, P. (2016). MEP aplica curva de 5 puntos en bachillerato de Matemática. La prensa libre.cr. Recuperado de: https://www.laprensalibre.cr/Noticias/detalle/69811/mep-aplica-curvade-5-puntos-en-bachillerato-de-matematica-

Saavedra, A. (2011). Diseño e implementación de ambientes virtuales de aprendizaje a través de la construcción de un curso virtual en la asignatura de química para estudiantes de grado 11 de la institución educativa José Asunción Silva (Tesis de maestría). Universidad Nacional de Colombia, Palmira. Recuperado de: http://www.bdigital.unal.edu.co/6129/1/albaluciasaavedraabadia.2011.pdf

Saldaña, O., Escartín, J., Peña, J., Jiménez, Y., Ceja, L., Rey, A., Vidal, T., \& Rodríguez, A. (2014). Fortalezas personales relacionadas con el rendimiento académico y profesional en el campo de la psicología social y la psicología social aplicada. En I Congrés Internacional d'Educació Emocional. Recuperado de: http://diposit.ub.edu/dspace/bitstream/2445/58743/1/C150_162.pdf

Ulloque, R. (2016). Aplicación del Uso del Software eXelearning y el Desarrollo de Capacidades para la Producción de Textos en Estudiantes de Secundaria. Universidad de San Martin de Porres, Lima. Recuperado de: http://www.repositorioacademico.usmp.edu.pe/bitstream/usmp/2327/1/ulloque_drv.pdf 